\title{
Influência da interação genótipo $x$ ambiente sobre o peso à desmama em bovinos da raça Nelore
}

\author{
[Influence of genotype x environment interaction on weaning weight in Nellore cattle] \\ S. Ribeiro ${ }^{1}$, J.P. Eler ${ }^{2}$, J.C.C. Balieiro ${ }^{2}$, J.B.S. Ferraz ${ }^{2}$, V.B. Pedrosa ${ }^{1}$, E.C. Mattos ${ }^{1}$ \\ ${ }^{1}$ Aluno de pós-graduação - FZEA-USP - Pirassununga, SP \\ ${ }^{2}$ Faculdade de Zootecnia e Engenharia de Alimentos - USP \\ Caixa Postal 23 \\ 13630-970 - Pirassununga, SP
}

\begin{abstract}
RESUMO
Avaliaram-se os efeitos da interação genótipo x ambiente sobre a característica peso à desmama em bovinos da raça Nelore. Foram analisados 45.697 registros de peso à desmama ajustados para 205 dias, originários de três fazendas. Estas foram organizadas em duplas e analisadas por meio de análises de características únicas, considerando cada dupla como um só rebanho. Em seguida, as mesmas duplas foram submetidas a análises de características múltiplas, em que o peso à desmama de cada fazenda foi considerado como característica distinta dentro de sua respectiva dupla. Os componentes de variância foram estimados por máxima verossimilhança restrita, utilizando-se o programa MTDFREML. Os coeficientes de correlação genética encontrados variaram de 0,79 a 1,00. Foram também estimadas as diferenças esperadas de progênies (DEPs), utilizando-se o mesmo programa computacional. Posteriormente, os touros foram ordenados conforme os valores de suas respectivas DEPs, selecionandose os 30\% mais bem classificados. Com base nas DEPs, estimou-se a correlação momento-produto de Pearson para os animais selecionados em dois diferentes ambientes, de modo a verificar as diferenças de ordenação. As correlações de Pearson estenderam-se de 0,96 a 0,99 , sugerindo pequeno ou nenhum efeito da interação genótipo $\mathrm{x}$ ambiente nos rebanhos incluídos neste estudo.
\end{abstract}

Palavras-chave: meio ambiente, bovinos de corte, genótipo, parâmetros genéticos, peso à desmama

\begin{abstract}
The effects of genotype $x$ environment interaction on weaning weight in Nellore cattle were evaluated. A total of 45,697 records of weaning weight adjusted for 205 days obtained from three farms were analyzed. The farms were organized in pairs and analyzed by single-trait analysis, as if they were only one herd. Afterwards, the pairs were submitted to multiple-trait analyses, once the same trait was considered as a distinct trait in each farm. The variance components were estimated by Restricted Maximum Likelihood, using the program MTDFREML. The genetic correlation coefficients varied from 0.79 to 1.00. The Expected Progeny Differences (EPD) were estimated using de same computational program. Later, the sires were organized in accordance with their EPD, selecting the $30 \%$ best classified sires. Pearson's correlations were estimated among the EPD of animals selected in two different environments, in order to verify changes in their classification. The Pearson's correlations extended from 0.96 to 0.99, suggesting small or inexistent effect of genotype $x$ environment interaction in the herds included in this study.
\end{abstract}

Keywords: beef cattle, environment, genotype, genetic parameters, weaning weight

Recebido em 4 de março de 2008

Aceito em 5 de março de 2009

E-mail: sandraribeiro@usp.br 


\section{INTRODUÇÃO}

Nos principais programas de melhoramento genético de bovinos de corte em execução no Brasil, a pressuposição comumente assumida nas avaliações genéticas é a ausência de interação genótipo $\mathrm{x}$ ambiente, ou seja, as variâncias residuais e genéticas são consideradas constantes para todos os rebanhos participantes. Neste caso, são desconsiderados os níveis de produção e as variâncias particulares dos diferentes ambientes avaliados. De acordo com Van Vleck (1987), se as variâncias genéticas e residuais e as covariâncias fossem conhecidas em cada ambiente, a seleção com base nos resultados obtidos das análises de características múltiplas em modelos mistos produziria uma avaliação com maior acurácia.

Falconer (1952) introduziu o conceito da correlação genética entre desempenhos em ambientes diferentes, usando a taxa de resposta indireta (correlacionada), em relação à resposta direta, para determinar o ambiente ótimo para seleção. Se a correlação genética for alta, então o desempenho em dois ambientes representará aproximadamente o mesmo caráter, determinado pelo mesmo grupo de genes.

A preocupação sobre respostas dos indivíduos em função dos diferentes ambientes em que são avaliados tem crescido em estudos de características de crescimento. Conforme mencionado por Eler et al. (2000), as acurácias das estimativas de valor genético em diferentes ambientes são reduzidas caso essas interações forem importantes. A menos que o touro tenha progênies em muitos ambientes, qualquer efeito da interação seria confundido com a estimativa de mérito genético.

Análises de dados de campo têm indicado a presença de correlações genéticas significativas entre informações de desempenho dos animais em diferentes ambientes. Toral et al. (2004) observaram este efeito sobre as características peso ao nascimento, aos 205, aos 365 e aos 550 dias em bovinos Nelore provenientes de três regiões do Estado de Mato Grosso do Sul. Neser et al. (1998) encontraram evidências da ação da interação genótipo $\mathrm{x}$ ambiente sobre o peso à desmama em bovinos Bonsmara criados em três regiões da África do Sul. Lee e Bertrand (2002) observaram que o ganho de peso pós-desmama mostrou ser afetado pelo efeito desta interação em animais da raça Hereford, quando avaliados na Argentina, Canadá, Uruguai e Estados Unidos. Resultados semelhantes foram encontrados por Ferreira et al. (2001) na raça Nelore.

A interação genótipo $\mathrm{x}$ ambiente, quando não é devidamente detectada, implica predição viesada dos valores genéticos, com consequente redução do progresso genético. Pelo exposto, este estudo teve por objetivos avaliar o efeito da interação genótipo $\mathrm{x}$ ambiente sobre parâmetros genéticos da característica peso à desmama em bovinos Nelore, bem como sobre o comportamento da classificação dos reprodutores.

\section{MATERIAL E MÉTODOS}

Os dados foram obtidos junto ao Grupo de Melhoramento Animal (GMA) da Faculdade de Zootecnia e Engenharia de Alimentos (FZEA/USP), em Pirassununga, SP. Foram analisados 45.697 registros de peso à desmama originários de animais nascidos entre 1987 e 2002 e distribuídos em três fazendas (R1, R2 e R3) pertencentes à Agro-Pecuária CFM Ltda. As fazendas R1 (20¹7'62' S e 49 51'23'' W) e R3 (20³4'03', S e 50 $13^{\circ} 49^{\prime}$ ' W) estão localizadas na região oeste do estado de São Paulo, enquanto

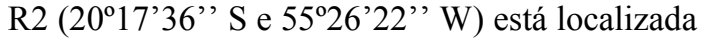
no estado de Mato Grosso do Sul. O sistema de criações exclusivamente em pastagens é adotado nas três fazendas do grupo, com suplementação de sal mineralizado à vontade. As pastagens que predominam nas fazendas são constituídas por braquiária (Brachiaria decumbens). Os grupos contemporâneos foram caracterizados por animais nascidos em mesmo rebanho-anoestação, de mesmo sexo e grupo de manejo.

O peso à desmama foi previamente ajustado para o efeito de idade à mensuração, utilizando-se a técnica de polinômios segmentados. Para a determinação dos segmentos iniciais que formaram a função, usou-se um diagrama de dispersão com o objetivo de identificar as regiões onde poderiam ocorrer as mudanças de comportamento da distribuição dos valores fenotípicos. As análises de regressão foram realizadas utilizando o procedimento NLIN do SAS/1995, para estimar os parâmetros e definir o polinômio segmentado quadrático-quadrático (Gallant e Fuller, 1973; Mourão et al., 2004). 
Para fins de comparação e melhor avaliação dos resultados, foram realizadas análises que consideram a interação genótipo $\mathrm{x}$ ambiente $\mathrm{e}$ análises que a desconsideram. Nas análises que desconsideram a interação genótipo $\mathrm{x}$ ambiente, as fazendas foram organizadas em duplas, ou seja, R1R2, R1R3 e R2R3. Estas foram avaliadas por meio de análises de características únicas, como se cada dupla formasse apenas um rebanho. No caso das avaliações que consideram a interação genótipo $\mathrm{x}$ ambiente, processaram-se análises de características múltiplas, utilizandose as mesmas duplas de fazendas, considerandose o peso à desmama em cada fazenda como uma característica distinta. Assim, o modelo utilizado pelas análises conjuntas da característica nas três fazendas foi o mesmo utilizado nas análises de característica única, mas considerando a distribuição multivariada e a diversidade entre as três fazendas no que se refere às variâncias residuais e genéticas.

A conectividade entre as fazendas foi definida por "touros de conexão", mantendo-se apenas aquelas cujas progênies estavam presentes simultaneamente nas duas fazendas constituintes da dupla analisada.

O modelo utilizado nas análises considerou o grupo contemporâneo e a classe de idade da mãe ao parto como efeitos fixos, além dos efeitos aleatórios genético direto, genético materno, efeito de ambiente permanente e efeito residual. O modelo é descrito a seguir:

$\mathrm{y}_{\mathrm{ijklmn}}=\mu+\mathrm{g}_{1}+\mathrm{p}_{\mathrm{ijk}}+\mathrm{m}_{\mathrm{ijk}}+\mathrm{c}_{\mathrm{m}}+\mathrm{a}_{\mathrm{ij}}+\mathrm{e}_{\mathrm{ijklmn}}$,

em que $\mathrm{y}_{\mathrm{ijklmn}}=$ peso à desmama ajustado para 205 dias do animal $\underline{n}$, da classe de idade da mãe ao parto $\underline{\mathrm{m}}$, pertencente ao grupo contemporâneo à desmama $\underline{1}$, no parto $\underline{\mathrm{k}}$ da matriz $\mathrm{i}$ e filho do reprodutor $\underline{i} ; \mu=$ constante inerente a todas as observações; $g_{1}=$ efeito fixo da classe de grupo contemporâneo à desmama $1 ; p_{i j k}=$ efeito aleatório de ambiente permanente, com média 0 e variância $\sigma_{\text {pe }}^{2} ; m_{i j k}=$ efeito aleatório aditivo materno, com média 0 e variância $\sigma_{\mathrm{m}}^{2} ; \mathrm{c}_{\mathrm{m}}=$ efeito fixo da classe de idade da mãe ao parto $\underline{\underline{m}}$; $\mathrm{a}_{\mathrm{ij}}=$ efeito aleatório aditivo direto, com média 0 e variância $\sigma_{\mathrm{a}}^{2} ; \mathrm{e}_{\mathrm{ijklmn}}=$ efeito aleatório residual associado ao peso à desmama ajustado para 205 dias do animal $\underline{n}$, da classe de idade da mãe ao parto $\underline{\mathrm{m}}$, pertencente ao grupo contemporâneo à desmama 1 , no parto $\mathrm{k}$ da matriz $\mathrm{j}$ e filho do reprodutor $\underline{\mathrm{i}}$, com média 0 e variância $\sigma_{\mathrm{e}}^{2}$.

A idade da mãe ao parto foi distribuída em oito classes (CIMP), sendo: CIMP $1=$ menor que 28 meses; CIMP $2=27$ a 37 meses; CIMP $3=36$ a 49 meses; CIMP $4=48$ a 73 meses; CIMP $5=$ 72 a 121 meses; CIMP $6=120$ a 145 meses; CIMP $7=144$ a 181 meses; CIMP $8=$ maior que 180 meses.

Foram utilizadas nas análises as matrizes de parentesco referentes aos pares de fazendas R1R2, R1R3 e R2R3, com pedigrees de 46.800, 53.174 e 27.922 animais, respectivamente. As soluções das equações de modelos mistos foram obtidas por máxima verossimilhança restrita, utilizando-se o programa MTDFREML (Boldman et al., 1995).

Com o propósito de verificar a relação de proporção entre os efeitos genéticos diretos e os efeitos residuais, foram calculadas razões entre os componentes de variância residual e os componentes de variância genética direta dentro de cada tipo de análise (análises de características únicas e múltiplas). O critério de convergência adotado foi aquele em que a variância do simplex atingiu $10^{-9}$, por pelo menos dois "recomeços", com os parâmetros estimados em rodada anterior, sem variação do $-2 \Lambda$ na sexta casa decimal.

A partir dos resultados obtidos nas análises anteriores, foram calculadas as DEPs dos reprodutores, as quais foram comparadas para verificar $\mathrm{o}$ impacto da interação genótipo $\mathrm{x}$ ambiente na avaliação genética e na classificação dos touros. Consideraram-se como reprodutores os machos nascidos até a safra de 2001, em que cada touro deveria ter progênies em pelo menos duas das três fazendas, com número mínimo de dois filhos (as) em cada uma destas fazendas.

Para os resultados das análises de características únicas, foram preparados arquivos contendo as DEPs dos reprodutores para cada dupla de fazendas, totalizando três arquivos: DEPs oriundas da análise de R1R2, DEPs de R1R3 e DEPs de R2R3. No caso dos resultados originados pelas análises de características múltiplas, foram organizados arquivos com as DEPs dos touros especificamente para cada fazenda formadora da dupla analisada. Assim, 
foram preparados seis arquivos: dois arquivos contendo as DEPs dos touros de R1, originados pela análise da dupla R1R2 e análise de R1R3; dois arquivos de DEPs dos touros de $\mathrm{R} 2$, originados pela análise das duplas R1R2 e R2R3; e dois arquivos de DEPs dos touros de R3, originados pela análise das duplas R1R3 e R2R3.

As DEPs dos touros contidas nos arquivos das fazendas individuais foram correlacionadas com as DEPs observadas nos arquivos das duplas de fazendas. Tal procedimento foi adotado para verificar possíveis ocorrências de alteração das DEPs conforme o tipo de análise a que os animais foram submetidos. $O$ método utilizado foi a correlação momento-produto de Pearson, utilizando o procedimento PROC CORR do programa SAS/1995. Posteriormente, foram selecionados e classificados os 30\% melhores reprodutores em cada análise, ou seja, dentro de cada dupla e de cada fazenda específica. A partir desta seleção, calcularam-se as porcentagens de animais selecionados em comum entre as duplas de fazendas e as fazendas individuais, além dos índices de discordância entre as classificações.

\section{RESULTADOS E DISCUSSÃO}

Os números de observações e as estimativas de médias, desvios-padrão, coeficientes de variação, mínimo e máximo para a característica peso à desmama dentro das respectivas fazendas avaliadas encontram-se na Tab. 1

As fazendas R1 e R3 apresentaram maiores médias em relação à fazenda R2. As medidas de variabilidade para a característica peso à desmama nas diferentes fazendas mostraram-se similares, com desvios-padrão oscilando de 22,20 a $22,46 \mathrm{~kg}$. Tal variação também foi verificada nos coeficientes de variação, os quais se estenderam de $11,2 \%$ a 12,4\%. Na Tab. 2, são apresentadas as estimativas dos componentes de (co)variância obtidas nas análises de características únicas para a característica peso à desmama nas fazendas organizadas em duplas.

Tabela 1. Números de observações (N), médias (MED), desvios-padrão (DP), coefícientes de variação, mínimo (MIN) e máximo (MAX) para a característica peso à desmama, segundo a fazenda de mensuração (em $\mathrm{kg}$ )

\begin{tabular}{ccccccc}
\hline Fazenda & $\mathrm{N}$ & MED & DP & CV $(\%)$ & MIN & MAX \\
\hline R1 & 10.662 & 196,70 & 22,46 & 11,42 & 125,70 & 256,30 \\
R2 & 8.947 & 181,24 & 22,41 & 12,36 & 125,10 & 254,80 \\
R3 & 26.088 & 198,77 & 22,20 & 11,17 & 125,10 & 256,30 \\
\hline
\end{tabular}

Tabela 2. Número de observações, estimativas dos componentes de variância genética aditiva direta $\left(\hat{\sigma}_{a}^{2}\right)$, materna $\left(\hat{\sigma}_{m}^{2}\right)$, residual $\left(\hat{\sigma}_{e}^{2}\right)$ e fenotípica $\left(\hat{\sigma}_{p}^{2}\right)$, componentes de covariância entre os efeitos genético aditivo direto e materno $\left(\hat{\sigma}_{a, m}\right)$, coeficientes de herdabilidade direta $\left(\hat{h}_{a}^{2}\right)$ e materna $\left(\hat{h}_{m}^{2}\right)$ e correlação entre efeitos genético aditivo direto e materno $\left(\hat{r}_{a, m}\right)$ para a característica peso à desmama (P205) nas duplas de fazendas

R1R2, R1R3 e R2R3, obtidas em análises de características únicas

\begin{tabular}{cccccccccc}
\hline Fazendas & $\mathrm{N}$ & $\hat{\sigma}_{a}^{2}$ & $\hat{\sigma}_{m}^{2}$ & $\hat{\sigma}_{a, m}$ & $\hat{\sigma}_{e}^{2}$ & $\hat{\sigma}_{p}^{2}$ & $\hat{h}_{a}^{2}$ & $\hat{h}_{m}^{2}$ & $\hat{r}_{a, m}$ \\
\hline R1R2 $^{\text {a }}$ & 19.609 & 80,14 & 19,07 & $-3,50$ & 169,93 & 314,09 & 0,26 & 0,06 & $-0,09$ \\
R1R3 $^{\text {b/ }}$ & 36.750 & 108,04 & 12,31 & 2,34 & 153,32 & 325,62 & 0,33 & 0,04 & 0,06 \\
R2R3 $^{\text {c/ }}$ & 35.035 & 89,15 & 13,00 & 9,50 & 165,53 & 320,62 & 0,28 & 0,04 & 0,28 \\
\hline
\end{tabular}

a/ Análises de característica única para a característica P205 nas fazendas R1 e R2.

b/Análises de característica única para a característica P205 nas fazendas R1 e R3.

c/Análises de característica única para a característica P205 nas fazendas R2 e R3.

As relações entre os componentes de variância residual e genética aditiva direta foram de 2,12 para R1R2, 1,42 para R1R3 e de 1,86 para R2R3. As estimativas dos coeficientes de herdabilidade direta de cada dupla acompanharam o comportamento de suas respectivas relações acima descritas; os coeficientes de herdabilidade foram de 0,26, 0,33 e
0,28 para as duplas R1R2, R1R3 e R2R3, respectivamente. As estimativas dos componentes de (co)variância para a característica peso à desmama obtidas nas três fazendas por meio de análises de características múltiplas encontram-se na Tab. 3. 
Tabela 3. Estimativas dos componentes de variância genética aditiva direta $\left(\hat{\sigma}_{a}^{2}\right)$, materna $\left(\hat{\sigma}_{m}^{2}\right)$, residual $\left(\hat{\sigma}_{e}^{2}\right)$, fenotípica $\left(\hat{\sigma}_{p}^{2}\right)$ e de ambiente permanente $\left(\hat{\sigma}_{p e}^{2}\right)$, componentes de covariância entre os efeitos genéticos aditivos diretos da primeira e da segunda fazendas constituintes da dupla analisada $\left(\hat{\sigma}_{a, a}\right)$, componentes de covariância entre os efeitos maternos da primeira e da segunda fazendas constituintes da dupla analisada $\left(\hat{\sigma}_{m, m}\right)$ e componentes de covariância entre efeitos genético aditivo direto e materno dentro das fazendas $\left(\hat{\sigma}_{a, m}\right)$ para a característica peso à desmama (P205) nas fazendas R1, R2 e R3, obtidas em análises de características múltiplas

\begin{tabular}{|c|c|c|c|c|c|c|c|c|}
\hline Fazendas & $\hat{\sigma}_{a}^{2}$ & $\hat{\sigma}_{m}^{2}$ & $\hat{\sigma}_{a, a}$ & $\hat{\sigma}_{m, m}$ & $\hat{\sigma}_{a, m}$ & $\hat{\sigma}_{p e}^{2}$ & $\hat{\sigma}_{e}^{2}$ & $\hat{\sigma}_{p}^{2}$ \\
\hline $\mathrm{R}^{\mathrm{a}^{\mathrm{a}}}$ & 97,62 & 18,54 & 56,40 & 18,64 & $-13,54$ & 60,27 & 155,21 & 318,11 \\
\hline $\mathrm{R} 2^{\mathrm{a}^{/}}$ & 51,97 & 21,55 & --- & --- & 12,09 & 27,82 & 192,16 & 305,61 \\
\hline $\mathrm{R} 1^{\mathrm{b} /}$ & 106,96 & 6,97 & 99,96 & 8,50 & $-5,70$ & 62,12 & 150,22 & 320,58 \\
\hline $\mathrm{R} 3^{\mathrm{b} /}$ & 107,89 & 10,82 & --- & -- & 6,46 & 47,30 & 154,26 & 326,72 \\
\hline $\mathrm{R} \mathrm{c}^{\prime}$ & 64,51 & 25,80 & 73,47 & 14,00 & 5,30 & 26,70 & 186,18 & 308,49 \\
\hline $\mathrm{R}^{\mathrm{c}^{-/}}$ & 101,94 & 13,90 & --- & --- & 6,84 & 45,65 & 187,19 & 325,51 \\
\hline
\end{tabular}

a/ Análises de características múltiplas para a característica P205 nas fazendas R1 e R2.

b/ Análises de características múltiplas para a característica P205 nas fazendas R1 e R3.

c/ Análises de características múltiplas para a característica P205 nas fazendas R2 e R3.

As estimativas de herdabilidade direta e materna e coeficientes de correlação genética entre os efeitos genéticos diretos e maternos para o peso à desmama obtidas em análises de características múltiplas estão descritas na Tab. 4.
Com exceção da fazenda R2, os coeficientes de herdabilidade nas fazendas avaliadas, por meio das análises de características múltiplas, mantiveram-se próximos daqueles obtidos nas análises de características únicas.

Tabela 4. Estimativas de coeficientes de herdabilidade direta $\left(\hat{h}_{a}^{2}\right)$ e materna $\left(\hat{h}_{m}^{2}\right)$, coeficientes de correlação entre os efeitos genético aditivo direto e materno dentro das fazendas $\left(\hat{r}_{a, m}\right)$ e coeficientes de correlação entre os efeitos genéticos aditivos diretos entre as fazendas constituintes da dupla analisada ( $\hat{r}_{a, a}$ ) para a característica peso à desmama (P205), nas fazendas R1, R2 e R3, obtidas em análises de características múltiplas

\begin{tabular}{|c|c|c|c|c|}
\hline Fazendas & $\hat{h}_{a}^{2}$ & $\hat{h}_{m}^{2}$ & $\hat{r}_{a, m}$ & $\hat{r}_{a, a}$ \\
\hline $\mathrm{R} 1^{\mathrm{a}}{ }^{\prime}$ & 0,31 & 0,06 & $-0,32$ & \\
\hline $\mathrm{R} 2^{\mathrm{a} /}$ & 0,17 & 0,07 & 0,36 & 0,79 \\
\hline $\mathrm{R} 1 \mathrm{~b}^{-}$ & 0,33 & 0,02 & $-0,21$ & \\
\hline $\mathrm{R} 3^{\mathrm{b} /}$ & 0,33 & 0,03 & 0,19 & 0,93 \\
\hline $\mathrm{R} 2^{\mathrm{c} /}$ & 0,21 & 0,08 & 0,13 & \\
\hline $\mathrm{R} 3^{\text {c/ }}$ & 0,31 & 0,04 & 0,18 & 0,91 \\
\hline
\end{tabular}

al Análises de características múltiplas para a característica P205 nas fazendas R1 e R2.

b/ Análises de características múltiplas para a característica P205 nas fazendas R1 e R3.

c/ Análises de características múltiplas para a característica P205 nas fazendas R2 e R3.

Pela análise dos resultados, observou-se que, quando a análise incluía a fazenda R2, houve decréscimo dos coeficientes de herdabilidade direta nas fazendas R1 e R3. Por outro lado, foi constatado aumento das estimativas de herdabilidade materna nas fazendas R1 e R3 quando estas foram avaliadas em conjunto com a fazenda R2. O efeito genético materno é definido como o efeito dos genes da mãe do indivíduo, condicionando o ambiente em que esta provê seu filho durante a gestação e na fase de aleitamento. A quantificação dessa forma indireta de expressão gênica é passível de sofrer influência do ambiente. Progênies criadas na fase prédesmama em ambientes menos favoráveis possivelmente fornecerão maiores oportunidades 
para a expressão do efeito materno sobre seu desenvolvimento, o que será expresso na forma de maiores estimativas de variância genética materna e herdabilidade materna. Dentro desse conceito, possivelmente por um ambiente menos propício ao crescimento dos animais, a fazenda R2 seria causadora de alterações no comportamento das estimativas dos efeitos direto e materno nas fazendas R1 e R3, quando a análise incluía R2.

As estimativas de herdabilidade direta encontradas para o peso à desmama neste trabalho estão próximas dos valores obtidos por Souza et al. (1998), que constataram estimativas de herdabilidade de 0,25 a 0,39, e também por Eler et al. (1996), os quais verificaram a estimativa de 0,29 para este mesmo parâmetro na raça Nelore.

As correlações entre as estimativas dos componentes de variância genéticos aditivos obtidos neste estudo variaram de 0,79 a 0,93 , os quais, de acordo com o descrito por Robertson (1959), não seriam indicadores de presença nítida da interação genótipo $\mathrm{x}$ ambiente sobre a característica estudada.

As correlações de Pearson estenderam-se de 0,96 a 0,99 , valores próximos dos encontrados por Dodenhoff et al. (1999), na raça Angus, e por Lee e Pollak (1997), na raça Simental. Ambos obtiveram valor igual a 0,97 para a correlação. Por outro lado, as estimativas encontradas neste trabalho foram mais altas que as observadas por Toral et al. (2004) na raça Nelore. Para esses autores, as correlações estenderam-se de 0,86 a 0,89 .

As altas estimativas de correlação de Pearson observadas entre as DEPs dos touros para a característica em estudo são um indicador de que estes seriam classificados de maneira semelhante nas análises que desconsideram a interação genótipo $\mathrm{x}$ ambiente (na qual as fazendas são analisadas como apenas um rebanho em análises de características únicas) e naquelas que consideram este tipo de interação (em que as fazendas são analisadas como características distintas em análises de características múltiplas). Dentre os $30 \%$ melhores touros selecionados para o peso à desmama, a porcentagem de indivíduos comuns entre as duplas de fazendas e as fazendas específicas variou de 86 a $97,7 \%$.

Os coeficientes de correlação de Pearson entre as DEPs dos reprodutores para peso à desmama podem ser observados na Tab. 5 .

A relação do número de touros classificados dentro da faixa percentual dos 30\% melhores, bem como a porcentagem de animais selecionados nas fazendas individuais não comuns aos classificados nas duplas de fazendas (índices de discordância) são apresentados na Tab. 6 .

Tabela 5. Coeficientes de correlação de Pearson entre as DEPs dos reprodutores para a característica peso à desmama (P205) obtidas a por meio de análises de características únicas e análises de características múltiplas

\begin{tabular}{|c|c|c|c|}
\hline & $\mathrm{R} 1 \mathrm{R} 2^{\mathrm{a}}$ & $\mathrm{R} 1^{\mathrm{b} /}$ & $\mathrm{R} 2^{-\mathrm{b}}$ \\
\hline $\mathrm{R}^{2} 2^{\mathrm{a}^{\prime}}$ & 1,00 & 0,96 & 0,96 \\
\hline $\mathrm{R} 1^{\mathrm{b} /}$ & 0,96 & 1,00 & 0,89 \\
\hline \multirow[t]{2}{*}{$\mathrm{R} 2^{\mathrm{b} /}$} & 0,96 & 0,89 & 1,00 \\
\hline & $\mathrm{R}^{\prime R} 3^{\mathrm{c}^{\prime}}$ & $\mathrm{R} 1^{\mathrm{d} /}$ & $\mathrm{R}^{\mathrm{d} /}$ \\
\hline $\mathrm{R} \mathrm{R} 3^{{ }^{\mathrm{c} /}}$ & 1,00 & 0,98 & 0,99 \\
\hline $\mathrm{R} 1^{\mathrm{d} /}$ & 0,98 & 1,00 & 0,97 \\
\hline \multirow[t]{2}{*}{$\mathrm{R} 3^{\mathrm{d} /}$} & 0,99 & 0,97 & 1,00 \\
\hline & $\mathrm{R}^{2} 3^{-\mathrm{e}^{\prime}}$ & $\mathrm{R} 2^{\mathrm{f} /}$ & $\mathrm{R} 3^{\mathrm{f} /}$ \\
\hline R2R3 ${ }^{\mathrm{e}}$ & 1,00 & 0,98 & 0,99 \\
\hline $\mathrm{R} 2^{\mathrm{f} /}$ & 0,98 & 1,00 & 0,96 \\
\hline $\mathrm{R} 3{ }^{\mathrm{f} /}$ & 0,99 & 0,96 & 1,00 \\
\hline
\end{tabular}

\footnotetext{
a/ Análise de características únicas para a característica P205 nas fazendas R1 e R2.

b/ Análises de características múltiplas para a característica P205 nas fazendas R1 e R2.

c/ Análise de características únicas para a característica P205 nas fazendas R1 e R3.

d/ Análises de características múltiplas para a característica P205 nas fazendas R1 e R3.

e/ Análise de características únicas para a característica P205 nas fazendas R2 e R3.

f/ Análises de características múltiplas para a característica P205 nas fazendas R2 e R3.
} 
Tabela 6. Número total de reprodutores e número de reprodutores incluídos no grupo dos $30 \%$ selecionados dentro das duplas de fazendas R1R2, R1R3 e R2R3, bem como os índices de discordância (porcentagens de indivíduos classificados nas fazendas individuais não comuns aos classificados nas duplas de fazendas) para a característica peso à desmama

\begin{tabular}{|c|c|c|c|c|c|}
\hline Fazendas & Total & Selecionados & $\mathrm{R} 1^{\mathrm{d} /}$ & $\mathrm{R} 2^{\mathrm{e}}-$ & $\mathrm{R} 3^{\mathrm{f} /}$ \\
\hline R1R2 ${ }^{a} /$ & 335 & 100 & $14,00 \%$ & $11,00 \%$ & - \\
\hline $\mathrm{R} 1 \mathrm{R} 33^{\mathrm{b}}$ / & 406 & 122 & $6,56 \%$ & - & $2,28 \%$ \\
\hline R2R3 $3^{\mathrm{c} /}$ & 410 & 123 & - & $8,94 \%$ & $4,07 \%$ \\
\hline
\end{tabular}

a/ DEPs obtidas por análises de características únicas na dupla de fazendas R1R2.

b/ DEPs obtidas por análises de características únicas na dupla de fazendas R1R3.

c/ DEPs obtidas por análise de características únicas na dupla de fazendas R2R3.

d/ DEPs da fazenda R1 obtidas por meio de análises de características múltiplas entre as fazendas R1R2 e/ou R1R3.

e/ DEPs da fazenda R2 obtidas por meio de análises de características múltiplas entre as fazendas R1R2 e/ou R2R3.

${ }^{\mathrm{f} /}$ DEPs da fazenda R3 obtidas por meio de análises de características múltiplas entre as fazendas R1R3 e/ou R2R3.

Considerando os touros selecionados dentro das fazendas individuais e das duplas de fazendas, 14 touros selecionados em R1 e 11 selecionados em R2 não estavam presentes dentre os 100 melhores touros classificados da dupla R1R2. No caso da dupla de fazendas R1R3, o número de touros não comuns dentre os 122 selecionados foi de oito indivíduos classificados na fazenda R1 e três da fazenda R3. Dentre os 123 touros classificados da dupla R2R3 não estavam presentes 11 touros selecionados em R2 e cinco touros selecionados em R3. A alteração da ordem dos animais classificados pode ser considerada um indício da influência da interação genótipoambiente sobre a predição das DEPs dos animais.

\section{CONCLUSÕES}

Nas três fazendas estudadas, os efeitos dos genes que controlam o peso à desmama em uma região coincidem, em sua maior parte, com aqueles que o controlam em outra região. Para este caso em especial, pode-se afirmar que, se a diferença entre os ambientes em que os animais foram avaliados for desconsiderada, o progresso genético mediante seleção não sofreria efeito significativo.

\section{REFERÊNCIAS BIBLIOGRÁFICAS}

BOLDMAN, K.G.; KRIESE, L.A.; VAN VLECK, L.D. et al. A manual for use of MTDFREML: a set of program to obtain estimates of variances and covariances (DRAFT). Lincoln: Department of Agriculture, Agricultural Research Service, 1995.
DODENHOFF, J.; VAN VLECK, L.D.; WILSON, D.E. Comparison of models to estimate genetic effects for weaning weight of Angus cattle. J. Anim. Sci., v.77, p.3176-3184, 1999.

ELER, J.P.; FERRAZ, J.B.S.; GOLDEN, B.L. et al. Influência da interação touro $\mathrm{x}$ rebanho na estimação da correlação entre efeitos genéticos direto e materno em bovinos da raça Nelore. Rev. Bras. Zootec., v.29, p.1642-1648, 2000.

ELER，J.P.; FERRAZ，J.B.S.; SILVA，P.R. Parâmetros genéticos para peso, avaliação visual e circunferência escrotal na raça Nelore, estimados por modelo animal. Arq. Bras. Med. Vet. Zootec., v.48, p.203-213, 1996.

FALCONER, D.S. The problem of environment and selection. Am. Nat., v.86, p.293-298, 1952.

FERREIRA, V.C.P.; PENNA, V.M.; BERGMANN, J.A.G. et al. Interação genótipoambiente em algumas características produtivas de gado de corte no Brasil. Arq. Bras. Med. Vet. Zootec., v.53, p.385-392, 2001.

GALLANT, A.R.; FULLER, W.A. Fitting segmented polynomial regression models whose join points have to be estimated. J. Am. Stat. Assoc., v.68, p.140-147, 1973.

LEE, C.; POLLAK, E.J. Relationship between sire $\mathrm{x}$. year interactions and direct-maternal genetic correlation for weaning weight of Simmental cattle. J. Anim. Sci., v.75, p.68-75, 1997.

LEE, D.H.; BERTRAND, J.K. Investigation of genotype $\mathrm{x}$ country interactions for growthtraits 
in beef cattle. J. Anim. Sci., v. 80, p.330-337, 2002.

MOURÃO, G.B.; MATTOS, E.C.; BALIEIRO, J.C.C. et al. Uso de polinômio segmentado para ajuste do peso à desmama da raça Nelore e seu efeito sobre as estimativas de parâmetros genéticos. In: REUNIÃO ANUAL DA SOCIEDADE BRASILEIRA DE ZOOTECNIA, 41., 2004, Campo Grande. Anais... Campo Grande: SBZ, 2004. CD-ROM (Melhoramento).

NESER, F.W.C.; ERASMUS, G.J.; WYK, J.B. Quantifying herd-year-season $\mathrm{x}$ sire interaction in Bonsmara cattle. S. Afr. J. Anim. Sci., v.28, p.167-172, 1998.

ROBERTSON, A. The sampling variance of the genetic correlation coefficient. Biometsics, v.15, p.469-485, 1959.
SOUZA, J.C.; RAMOS, A.A.; SILVA, L.O.C. et al. Effect of genotype $\mathrm{x}$ environment interaction on weaning weight of nellore calves raised in four different regions of Brazil. In: WORLD CONGRESS ON GENETICS APPLIED TO LIVESTOCK PRODUCTION, 6., 1998, Armidale, Austrália. Proceedings... Armidale: WCGALP, 1998. CD-ROM.

TORAL, F.L.B.; SILVA, L.O.C.; MARTINS, E.N. et al. Interação genótipo $\mathrm{x}$ ambiente em características de crescimento em bovinos da raça Nelore no Mato Grosso do Sul. Rev. Bras. Zootec., v.33, p.1445-1455, 2004.

VAN VLECK, L.D. Selection when traits have different genetic and phenotypic variances in different environments. J. Dairy Sci., v.70, p.337-344, 1987. 